\title{
Improving bounds on the order of regular graphs of girth 5
}

\author{
E. Abajo ${ }^{a}$, C. Balbuena ${ }^{\text {b,* }}$, M. Bendala ${ }^{a}$, X. Marcote ${ }^{b}$ \\ ${ }^{a}$ Departamento de Matemática Aplicada I, Universidad de Sevilla, Spain \\ b Departament d'Enginyeria Civil i Ambiental, Universitat Politècnica de Catalunya, E-08034 Barcelona, Spain
}

\section{A R T I C L E I N F O}

\section{Article history:}

Received 14 April 2018

Accepted 8 February 2019

Available online $\mathrm{xxxx}$

\section{Keywords:}

Small regular graphs

Cage

Girth

Amalgam

Elliptic semiplane

\begin{abstract}
A B S T R A C T
A $(k, g)$-graph is a $k$-regular graph with girth $g$ and a $(k, g)$-cage is a $(k, g)$-graph with the fewest possible number of vertices $n(k, g)$. Constructing $(k, g)$-cages and determining the order are both very hard problems. For this reason, an intensive line of research is devoted to constructing smaller $(k, g)$-graphs than previously known ones, providing in this way new upper bounds to $n(k, g)$ each time such a graph is constructed.

The paper focuses on girth $g=5$, where cages are known only for degrees $k \leq 7$. We construct $(k, 5)$-graphs using and extending techniques of amalgamation into the incidence graphs of elliptic semiplanes of type $\mathcal{L}$ introduced and exposed by Funk (2009). The order of these graphs provides better upper bounds on $n(k, 5)$ than those known so far, for values of $k$ such that either $13 \leq k \leq 33$ or $k \geq 66$.
\end{abstract}

CC 2019 Elsevier B.V. All rights reserved.

\section{Introduction}

All considered graphs are undirected and simple. For undefined terminology and notations the reader may refer to [5]. Let $G$ be a graph with vertex set $V(G)$ and edge set $E(G)$. If the vertex set is a multiplicative (additive) group, every edge $\{u, v\} \in E(G)$ has a Cayley color, defined as the pair $\left(u v^{-1}\right)^{ \pm 1}( \pm(u-v)$, respectively). The girth of a graph $G$ is the length $g$ of a shortest cycle. The set of vertices adjacent to a vertex $v \in V$ is denoted $N(v)$, and the degree of $v$ is the cardinality of $N(v)$.

$\mathrm{A}(k, g)$-graph is a $k$-regular graph of girth $g$ and a $(k, g)$-cage is a $(k, g)$-graph with the fewest possible number of vertices. Constructions of cages can be found in the complete survey [8]. The order of a $(k, g)$-cage is denoted by $n(k, g)$. A lower bound $n_{0}(k, g)$ on the number of vertices of a $(k, g)$-graph, known as the Moore bound, is given by:

$$
n_{0}(k, g)= \begin{cases}1+k+k(k-1)+\cdots+k(k-1)^{(g-3) / 2} & \text { if } g \text { is odd } \\ 2\left(1+(k-1)+\cdots+(k-1)^{g / 2-1}\right) & \text { if } g \text { is even. }\end{cases}
$$

In this paper we focus on the cage problem for $g=5$. In this case $n_{0}(k, 5)=1+k^{2}$, and this bound is only attained for $k=2,3,7$ and, perhaps, for $k=57$ (cf. [11]). The only known $(k, 5)$-graphs of minimum order for $k=4,5,6$ are cages (cf. $[13,14,18-21])$. No other $(k, 5)$-cage has been identified so far and most of the work carried out focus on constructing a new $(k, 5)$-graph with fewer vertices, for degrees $k>7$. Following the notation in [8,9], the currently best known bound on $n(k, 5)$ is given by the order $r e c(k, 5)$ of this graph.

The goal of this paper is to improve the bounds on $n(k, 5)$, that is, to find lower values of $\operatorname{rec}(k, 5)$. The methods are constructive, and based on the idea of amalgam. As far as we now, we reach this goal for most of the integers $k$ in the range $13, \ldots, 33$, for $k \in\{66,67,68\}$, and for every $k \geq 74$.

\footnotetext{
* Corresponding author.

E-mail addresses: eabajo@us.es (E. Abajo),m.camino.balbuena@upc.edu (C. Balbuena),mbendala@us.es (M. Bendala), francisco.javier.marcote@upc.edu (X. Marcote).
} 
Table 1

\begin{tabular}{lllll}
\multicolumn{5}{l}{ Upper bounds on the order of a $(k, 5)$-graph. } \\
\hline$k$ & Upper bound & Due to & Reference & New upper bound \\
\hline 8 & 80 & Royle & {$[15]$} & \\
9 & 96 & Jørgensen & {$[12]$} & \\
10 & 124 & Exoo & {$[7]$} & \\
11 & 154 & Exoo & {$[7]$} & \\
12 & 203 & Exoo & {$[7]$} & $\mathbf{2 2 6}$ \\
13 & 230 & Exoo & {$[7]$} & $\mathbf{2 8 0}$ \\
14 & 284 & Abreu et al. & {$[2]$} & \\
15 & 310 & Abreu et al. & {$[2]$} & \\
16 & 336 & Jørgensen & {$[12]$} & \\
17 & 436 & Abajo et al. & {$[1]$} & \\
18 & 468 & Abajo et al. & {$[1]$} & \\
19 & 500 & Abajo et al. & {$[1]$} & \\
20 & 564 & Abajo et al. & {$[1]$} & \\
21 & 666 & Abajo et al. & {$[1]$} & $\mathbf{6 5 8}$ \\
22 & 704 & Abajo et al. & {$[1]$} & \\
23 & 880 & Funk & {$[9]$} & $\mathbf{8 7 4}$ \\
24 & 924 & Funk & {$[9]$} & $\mathbf{9 2 0}$ \\
25 & 968 & Funk & {$[9]$} & $\mathbf{9 6 0}$ \\
26 & 1012 & Funk & {$[9]$} & $\mathbf{1 0 1 0}$ \\
27 & 1056 & Funk & {$[9]$} & $\mathbf{1 0 5 4}$ \\
28 & 1200 & Funk & {$[9]$} & $\mathbf{1 1 9 2}$ \\
29 & 1248 & Funk & {$[9]$} & $\mathbf{1 2 4 0}$ \\
30 & 1404 & Funk & {$[9]$} & $\mathbf{1 3 9 2}$ \\
31 & 1456 & Funk & {$[9]$} & $\mathbf{1 4 4 4}$ \\
32 & 1624 & Abajo et al. & {$[1]$} & $\mathbf{1 6 0 8}$ \\
33 & 1680 & Abajo et al. & {$[1]$} & $\mathbf{1 6 6 4}$ \\
\hline
\end{tabular}

In Section 2 we consider the incidence graph $\mathcal{L}_{q}$ of the elliptic semiplane of type $L$, and define a new amalgamation technique inspired by papers from Jørgensen [12], Funk [9] and Abreu et al. [2]. This technique requires both regular and bi-regular graphs to increase the degree of the resulting graph while removing vertices in flexible ways. We also recall a class of graphs introduced in [1] that will play a key role in our constructions.

In Section 3 we work with prime powers from $q=11$ to $q=29$. By using the new technique we construct $(k, 5)$-graphs, for $k \in\{13,14,21,23, \ldots, 33\}$, that provide new bounds on $n(k, 5)$. Table 1 shows the values of $r e c(k, 5)$ for $8 \leq k \leq 33$ and highlights our contributions.

In the rest of the paper we restrict ourselves to amalgams of $r_{q}$-regular graphs into $\mathcal{L}_{q}$. This has been done in [1] (cf. [1], Table 2 and Theorems 1.1,1.2) for $r_{q}=5,6$; and the achieved values of $\operatorname{rec}(k, 5)$ for $34 \leq k \leq 65$ and $69 \leq k \leq 73$ remain untouched.

In Section 4, (cf. Theorem 4.1 and Corollary 4.1), we deal with $q=61$ and prime powers $q \geq 71$. For each $q$ we find the best possible amalgam with $r_{q}$-regular graphs to prove that

$$
n\left(q+r_{q}, 5\right) \leq 2\left(q^{2}-1\right)
$$

for $7 \leq r_{q} \leq 12$. This way, we improve the value $\operatorname{rec}(k, 5)$ for $k \in\{66,67,68\}$ and from $k=74$ up to $k=479+12=491$.

This process, which first fixes $r_{q}$ and then determines the related prime powers $q$, could continue indefinitely. Now we search for an explicit expression of $r_{q}$ as an increasing function of $q$, similar to Jørgensen's bound (cf. [12], Corollary 19):

$$
n\left(q+\left\lfloor\frac{\sqrt{q-1}}{4}\right\rfloor, 5\right) \leq 2\left(q^{2}-1\right) \text { for an odd prime power } q .
$$

In Section 5 (cf. Theorems 5.1-5.3) we improve this result and obtain better values of $\operatorname{rec}(k, 5)$ for $k>491$. The proof of these theorems is based on the finite, relative and direct product difference sets provided by Singer [17], Bose [6] and Ganley [10], respectively.

\section{Constructions}

Using position matrices, inspired by the ones introduced by Abreu et al. in [3], the adjacency matrix of a projective plane was directly obtained in [4]. Furthermore, using also the position matrices of a family of Latin Squares, the adjacency matrix of the following bipartite graph $\mathcal{L}_{q}=(\mathbb{L}, \mathbb{P})$ was also obtained in Theorem 4.4. of [4].

Definition $2.1([4])$. Let $q \geq 2$ be a prime power and $\mathbb{F}_{q}$ a Galois field. Denote $\mathbb{F}_{q}^{*}=\mathbb{F}_{q} \backslash\{0\}$. Consider the sets $\mathbb{L}=$ $\left(\mathbb{F}_{q} \times \mathbb{F}_{q}^{*}\right) \cup\left(\{\infty\} \times \mathbb{F}_{q}^{*}\right)$ and $\mathbb{P}=\left(\mathbb{F}_{q} \times \mathbb{F}_{q}^{*}\right) \cup\left(\{\infty\} \times \mathbb{F}_{q}^{*}\right)$ and denote by $\ell[a, i], p(x, j)$, the elements of $\mathbb{L}$ and $\mathbb{P}$, respectively. The bipartite graph $\mathcal{L}_{q}=(\mathbb{L}, \mathbb{P})$ is defined as follows:

$$
\text { For } i \in \mathbb{F}_{q}^{*}: N(\ell[a, i])= \begin{cases}\left\{p(a+i j, j): j \in \mathbb{F}_{q}^{*}\right\} \cup\{p(\infty, i)\} & \text { if } a \in \mathbb{F}_{q} \\ \left\{p(x, i): x \in \mathbb{F}_{q}\right\} & \text { if } a=\infty\end{cases}
$$


This graph $\mathcal{L}_{q}$ is in fact the incidence graph of an elliptic semiplane of type $L$. It is obtained by removing from the projective plane a line together with all its points, and another point not belonging to that line together with all the lines incident to it. Notice that the graph $\mathcal{L}_{q}$ inherits the duality principle from the projective plane: any valid sentence in $\mathcal{L}_{q}$ still holds true when lines $\ell[a, i]$ and points $p(x, j)$ are interchanged. In particular, the following properties of the graph $\mathcal{L}_{q}$ have been proved in [4] and they play a fundamental role throughout the paper.

Proposition $2.1([4])$. Let $\mathcal{L}_{q}$ be the bipartite graph given in Definition 2.1 for a prime power $q \geq 2$. Denote $L_{a}=\left\{\ell[a, i]: i \in \mathbb{F}_{q}^{*}\right\}$ and $P_{x}=\left\{p(x, j): j \in \mathbb{F}_{q}^{*}\right\}$, for $a, x \in \mathbb{F}_{q} \cup\{\infty\}$. The graph $\mathcal{L}_{q}$ has the following properties:

(i) it is a q-regular graph of girth 6 with order $2\left(q^{2}-1\right)$;

(ii) its vertex set admits a partition $\mathbb{L} \cup \mathbb{P}$, where $\mathbb{L}=\bigcup_{a \in \mathbb{F}_{q} \cup\{\infty\}} L_{a}$ and $\mathbb{P}=\bigcup_{x \in \mathbb{F}_{q} \cup\{\infty\}} P_{x}$;

(iii) it has diameter 4 and any two distinct vertices either in $L_{a}$ or in $P_{x}$ are at distance 4 ;

(iv) each block $P_{x}$ is connected to each block $L_{a}$ by a perfect matching for $a, x \in \mathbb{F}_{q} \cup\{\infty\}$ when $x \neq a$; this matching is obtained from the adjacency rule according to Definition 2.1.

Property (iii) in Proposition 2.1 suggests the idea of adding new edges between vertices in the sets $L_{a}$ and $P_{x}$ of the graph $\mathcal{L}_{q}$ in order to increase its regularity, as long as the girth of the resulting graph is at least 5 . The Cayley colors $\left(u v^{-1}\right)^{ \pm 1}$ of the edges $\{u, v\}$ of a graph with vertex set $\mathbb{F}_{q}^{*}$ play a central role [1,2,9,12]. Funk introduces the following amalgamation technique:

Definition 2.2 ([9]). Given a prime power $q \geq 3$ and graphs $G_{L}, G_{P}$ with vertex set $\mathbb{F}_{q}^{*}$, the amalgamation of these graphs into $\mathcal{L}_{q}$ is the graph $\mathcal{L}_{q}\left(G_{L}, G_{P}\right)$ obtained by adding to the graph $\mathcal{L}_{q}$ the sets of edges $\left\{\left\{\ell[a, i], \ell\left[a, i^{\prime}\right]\right\}:\left\{i, i^{\prime}\right\} \in E\left(G_{L}\right)\right\}$ and $\left\{\left\{p(x, j), p\left(x, j^{\prime}\right)\right\}:\left\{j, j^{\prime}\right\} \in E\left(G_{P}\right)\right\}$, with $a, x \in \mathbb{F}_{q} \cup\{\infty\}$.

Definition 2.3 ([9]). Given a prime power $q \geq 3$ and an integer $r>0$, the pair of $r$-regular graphs $G_{L}$, $G_{P}$ with vertex set $\mathbb{F}_{q}^{*}$ and girth $g \geq 5$ is said suitable for amalgamation into $\mathcal{L}_{q}$, or simple suitable, if they have disjoint sets of Cayley colors in $\mathbb{F}_{q}^{*}$.

Jørgensen (cf. [12], Theorem 3) defines an equivalent construction and achieves the same result with a different terminology.

Theorem 2.1 ([9,12]). Given a prime power $q \geq 3$ and a pair of $r$-regular graphs $G_{L}, G_{P}$ suitable for amalgamation into $\mathcal{L}_{q}$, the graph $\mathcal{L}_{q}\left(G_{L}, G_{P}\right)$ is $(q+r)$-regular, has girth at least five and order $2\left(q^{2}-1\right)$.

Next, we provide a refinement of this technique that exploits the particularities of the perfect matchings between $L_{\infty}$ and $P_{x}(x \neq \infty)$, and between $P_{\infty}$ and $L_{a}(a \neq \infty)$, that lead us to use specific graphs $H_{L}, H_{P}$ in addition to the pair $G_{L}, G_{P}$. While in $[9,12] V\left(G_{L}\right)=V\left(G_{P}\right)=\mathbb{F}_{q}^{*}$, in the new technique $V\left(G_{L}\right), V\left(G_{P}\right), V\left(H_{L}\right), V\left(H_{P}\right) \subseteq \mathbb{F}_{q}^{*}$. This removal of vertices is the key to reduce the value $\operatorname{rec}(k, 5)$.

Definition 2.4. Given a prime power $q \geq 3$ and graphs $G_{L}, G_{P}, H_{L}, H_{P}$ such that $V\left(G_{L}\right), V\left(G_{P}\right), V\left(H_{L}\right), V\left(H_{P}\right) \subseteq \mathbb{F}_{q}^{*}$, the amalgamation of these graphs into $\mathcal{L}_{q}$ is the graph denoted $\mathcal{L}_{q}\left(G_{L}, G_{P}, H_{L}, H_{P}\right)$ with vertex set $V_{L} \cup V_{P}$ and edge set $E_{L} \cup E_{P} \cup E_{q}$, where $V_{L}=\bigcup_{a \in \mathbb{F}_{q}}\left\{\ell[a, i]: i \in V\left(G_{L}\right)\right\} \cup\left\{\ell[\infty, i]: i \in V\left(H_{L}\right)\right\}, V_{P}=\bigcup_{x \in \mathbb{F}_{q}}\left\{p(x, j): j \in V\left(G_{P}\right)\right\} \cup\left\{p(\infty, j): j \in V\left(H_{P}\right)\right\}$, and $E_{L}=\left(\bigcup_{a \in \mathbb{F}_{q}}\left\{\left\{\ell[a, i], \ell\left[a, i^{\prime}\right]\right\}:\left\{i, i^{\prime}\right\} \in E\left(G_{L}\right)\right\}\right) \cup\left\{\left\{\ell[\infty, i], \ell\left[\infty, i^{\prime}\right]\right\}:\left\{i, i^{\prime}\right\} \in E\left(H_{L}\right)\right\}, E_{P}=\left(\bigcup_{x \in \mathbb{F}_{q}}\left\{\left\{p(x, j), p\left(x, j^{\prime}\right)\right\}\right.\right.$ : $\left.\left.\left\{j, j^{\prime}\right\} \in E\left(G_{P}\right)\right\}\right) \cup\left\{\left\{p(\infty, j), p\left(\infty, j^{\prime}\right)\right\}:\left\{j, j^{\prime}\right\} \in E\left(H_{P}\right)\right\}, E_{q}=E\left(\mathcal{L}_{q}\left[V_{L} \cup V_{P}\right]\right)$, where $\mathcal{L}_{q}\left[V_{L} \cup V_{P}\right]$ denotes the subgraph of $\mathcal{L}_{q}$ induced by $V_{L} \cup V_{P}$.

We determine conditions on the graphs $G_{L}, G_{P}, H_{L}, H_{P}$ to ensure that the amalgam graph $\mathcal{L}_{q}\left(G_{L}, G_{P}, H_{L}, H_{P}\right)$ is $(q+r)$-regular with girth at least five.

Definition 2.5. Let $q \geq 3$ be a prime power, $r \geq 0$ be an integer and two partitions $\mathbb{F}_{q}^{*}=R_{L} \cup T_{L} \cup W_{L}=R_{P} \cup T_{P} \cup W_{P}$. The ordered set of graphs $\left(G_{L}, G_{P}, H_{L}, H_{P}\right)$ is $r$-suitable for amalgamation into $\mathcal{L}_{q}$, or simply $r$-suitable, if it satisfies the following properties:

(i) $V\left(G_{L}\right)=\mathbb{F}_{q}^{*} \backslash W_{L}=R_{L} \cup T_{L}$, where the vertices in $R_{L}$ and $T_{L}$ have degree $r+\left|W_{P}\right|$ and $r+\left|W_{P}\right|+1$, respectively.

$V\left(G_{P}\right)=\mathbb{F}_{q}^{*} \backslash W_{P}=R_{P} \cup T_{P}$, where the vertices in $R_{P}$ and $T_{P}$ have degree $r+\left|W_{L}\right|$ and $r+\left|W_{L}\right|+1$, respectively.

(ii) $H_{L}$ and $H_{P}$ are $r$-regular graphs with $V\left(H_{L}\right)=\mathbb{F}_{q}^{*} \backslash\left(W_{P} \cup T_{P}\right)=R_{P}$ and $V\left(H_{P}\right)=\mathbb{F}_{q}^{*} \backslash\left(W_{L} \cup T_{L}\right)=R_{L}$.

(iii) The graphs $G_{L}, G_{P}, H_{L}$ and $H_{P}$ have girth at least 5 .

(iv) $E\left(H_{L}\right) \cap E\left(G_{P}\right)=\emptyset, E\left(H_{P}\right) \cap E\left(G_{L}\right)=\emptyset$ and $G_{L}, G_{P}$ have disjoint sets of Cayley colors in $\mathbb{F}_{q}^{*}$.

In Fig. 1 we illustrate the partitions of $\mathbb{F}_{q}^{*}$ and the vertex sets of the graphs $G_{L}, G_{P}, H_{L}, H_{P}$. The eliminated sets are shown in dashed lines.

We are interested in the properties of the amalgam graph $\mathcal{L}_{q}\left(G_{L}, G_{P}, H_{L}, H_{P}\right)$. Suitability guarantees that it is regular and free from triangles and quadrilaterals. 


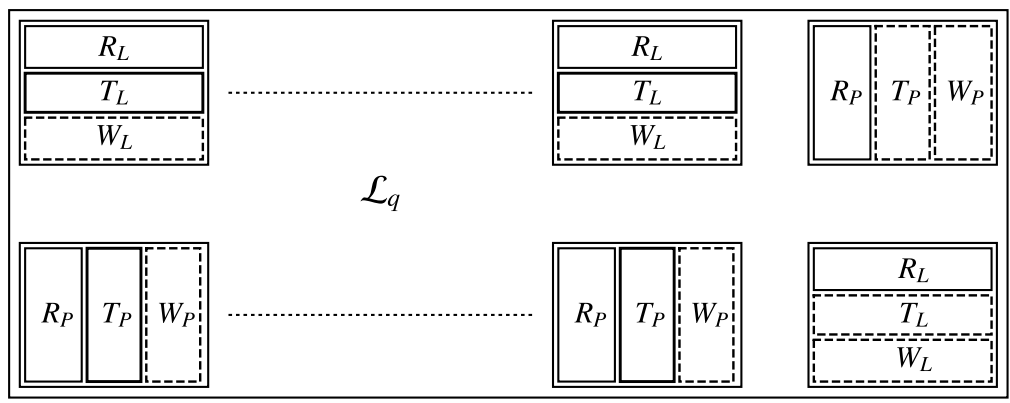

Fig. 1. $V\left(G_{L}\right)=R_{L} \cup T_{L}, V\left(G_{P}\right)=R_{P} \cup T_{P}, V\left(H_{L}\right)=R_{P}, V\left(H_{P}\right)=R_{L}$.

Theorem 2.2. Let $q \geq 3$ be a prime power, $r \geq 0$ be an integer, and $\left(G_{L}, G_{P}, H_{L}, H_{P}\right)$ be an ordered set of $r$-suitable graphs. The graph $\mathcal{L}_{q}\left(G_{L}, G_{P}, H_{L}, H_{P}\right)$ is $(q+r)$-regular, has girth at least 5 and order $2\left(q^{2}-1\right)-(q+1)\left(\left|W_{L}\right|+\left|W_{P}\right|\right)-\left(\left|T_{L}\right|+\left|T_{P}\right|\right)$.

Proof. Denote $G=\mathcal{L}_{q}\left(G_{L}, G_{P}, H_{L}, H_{P}\right)$ and $G^{*}=\mathcal{L}_{q}\left[V_{L} \cup V_{P}\right]$. Let us see that $G$ is $(q+r)$-regular by computing the degrees of the vertices of $V_{L}$, (the ones of $V_{P}$ should be treated in the same way).

In $G^{*}$ every vertex of $\bigcup_{a \in \mathbb{F}_{q}}\left\{\ell[a, i]: i \in V\left(G_{L}\right)\right\}$ has degree $q-1-\left|W_{P}\right|$ or $q-\left|W_{P}\right|$, depending on whether $i \in T_{L}$ or not, because

$$
N_{G^{*}}(\ell[a, i])= \begin{cases}\left\{p(a+i j, j): j \in \mathbb{F}_{q}^{*} \backslash W_{P}\right\} & \text { if } i \in T_{L} \\ \left\{p(a+i j, j): j \in \mathbb{F}_{q}^{*} \backslash W_{P}\right\} \cup p(\infty, i) & \text { if } i \notin T_{L}\end{cases}
$$

and every vertex in $\left\{\ell[\infty, i]: i \in V\left(H_{L}\right)\right\}$ remains with degree $q$, due to $N_{G^{*}}(\ell[\infty, i])=\left\{p(x, i): x \in \mathbb{F}_{q}\right\}$. From the definition of $G_{L}$ and $H_{L}$, the vertices of the set $V_{L}$ have degree $q+r$ in $G$ after amalgamation.

Let us check that $G$ has girth at least five. Denote by $C$ a shortest cycle of $G$ and suppose, by contradiction, that $|C| \leq 4$. The graph $G^{*}$ has girth at least 6 and the graphs $G_{L}, G_{P}, H_{L}, H_{P}$ have girth at least 5 , then the cycle $C$ must have at least an edge contained in $E(G) \backslash E\left(G^{*}\right)$. By Proposition 2.1(iv), a triangle cannot exist in $G$, and $C$ must be a cycle of length 4 . Suppose that $\left\{\ell[a, i], \ell\left[a, i^{\prime}\right]\right\}$ is an edge of $C$, for $a \in \mathbb{F}_{q}, i, i^{\prime} \in V\left(G_{L}\right)$ and $\left\{i, i^{\prime}\right\} \in E\left(G_{L}\right)$. Let $p(x, j) \in V(C)$ be a neighbor of $\ell[a, i]$ and $p\left(x, j^{\prime}\right) \in V(C)$ be a neighbor of $\ell\left[a, i^{\prime}\right]$, for $x \in \mathbb{F}_{q}$ and $j, j^{\prime} \in V\left(G_{P}\right)$. From Definition 2.1, it follows that $x=a+i j=a+i^{\prime} j^{\prime}$, yielding $i j=i^{\prime} j^{\prime}$ and $\left(j^{\prime} j^{-1}\right)^{ \pm 1}=\left(i i^{\prime-1}\right)^{ \pm 1}$. Since by hypothesis $G_{L}$ and $G_{P}$ share no Cayley color, it follows that $\left\{j, j^{\prime}\right\} \notin E\left(G_{P}\right)$; hence, $p(x, j)$ and $p\left(x, j^{\prime}\right)$ are not adjacent. Now assume that $\left\{\ell[\infty, i], \ell\left[\infty, i^{\prime}\right]\right\}$ is an edge of $C$, for $i, i^{\prime} \in V\left(H_{L}\right)$. From Definition 2.1, edges $\{\ell[\infty, i], p(x, i)\}$ and $\left\{\ell\left[\infty, i^{\prime}\right], p\left(x, i^{\prime}\right)\right\}$ belong to $E(G)$ for every $x \in \mathbb{F}_{q}$. Since $\left\{i, i^{\prime}\right\} \in E\left(H_{L}\right)$ and $E\left(H_{L}\right) \cap E\left(G_{P}\right)=\emptyset$, vertices $p(x, i)$ and $p\left(x, i^{\prime}\right)$ are not adjacent. The same argument holds if we suppose that $\left\{p(\infty, j), p\left(\infty, j^{\prime}\right)\right\}$ is an edge of $C$ with $j, j^{\prime} \in V\left(H_{P}\right)$. In any case, there is no cycle $C$ of length 4 in $G$.

Remark 2.1. Let $q \geq 3$ be a prime power and $r \geq 0$ be an integer. According to Theorem 2.2, the existence of $r$-suitable graphs $\left(G_{L}, G_{P}, H_{L}, H_{P}\right)$, for certain sets $W_{L}, W_{P}, T_{L}, T_{P} \subseteq \mathbb{F}_{q}^{*}$, ensures that

$$
n(q+r, 5) \leq 2\left(q^{2}-1\right)-(q+1)\left(\left|W_{L}\right|+\left|W_{P}\right|\right)-\left(\left|T_{L}\right|+\left|T_{P}\right|\right) .
$$

When $W_{L}=W_{P}=\emptyset$, the removal of $\gamma=1, \ldots, q$ sets $L_{a} \cup P_{a}$ from $\mathcal{L}_{q}\left(G_{L}, G_{P}, H_{L}, H_{P}\right)$, for $a \neq \infty$, provides

$$
n(q+r-\gamma, 5) \leq 2(q+1-\gamma)(q-1)-\left(\left|T_{L}\right|+\left|T_{P}\right|\right),
$$

and if $W_{L} \neq \emptyset$ or $W_{P} \neq \emptyset$, the deletion of a set $L_{a} \cup P_{a}$ for $a \neq \infty$ from $\mathcal{L}_{q}\left(G_{L}, G_{P}, H_{L}, H_{P}\right)$ produces a $(q+r-1, q+r)$-regular graph.

When $T_{L}=T_{P}=\emptyset$, the deletion of the set $L_{\infty} \cup P_{\infty}$ generates a graph which ensures that

$$
n(q+r-1,5) \leq 2 q(q-1)-q\left(\left|W_{L}\right|+\left|W_{P}\right|\right) .
$$

Remark 2.2. To simplify notation, we consider the natural isomorphism between the multiplicative group $\mathbb{F}_{q}^{*}$ and the additive group $\mathbb{Z}_{q-1}$, which allows us to identify $\xi^{u} \in \mathbb{F}_{q}^{*}$ with $u \in \mathbb{Z}_{q-1}$, for a primitive root $\xi \in \mathbb{F}_{q}^{*}$. Thus, we replace one group by the other in the following results. In particular, let us notice that the weight or Cayley color of an edge $\{u, v\}$ is $\pm(u-v)$, for vertices $u, v \in \mathbb{Z}_{q-1}$. By $\omega(G)$ we denote the set of Cayley colors of the edges of a graph $G$ whose vertex set $V(G) \subseteq \mathbb{Z}_{q-1}$.

Next we describe some graphs of girth at least 5 introduced in [1], which are very useful for constructing suitable graphs for amalgamation. 
Definition $2.6([1])$. Let $N \geq 5$ be an integer, and let $k_{1}, \ldots, k_{m}$ be a sequence of different odd elements from $\mathbb{Z}_{2 N}$.

(i) We denote by $S_{2 N}\left(k_{1}, \ldots, k_{m}\right)$ the graph with vertex set $\mathbb{Z}_{2 N}$ and edge set $\left\{\left\{2 v, 2 v+k_{j}\right\}: 0 \leq v \leq N-1,1 \leq j \leq m\right\}$ where the sum is performed modulo $2 N$.

(ii) Let $0<P, Q<N$ be two different even elements from $\mathbb{Z}_{2 N}$. We denote by $S_{2 N}\left(P, Q ; k_{1}, \ldots, k_{m}\right)$ the graph obtained by adding to $S_{2 N}\left(k_{1}, \ldots, k_{m}\right)$ the new edges $\{2 v, 2 v+P\}$ and $\{2 v+1,2 v+1+Q\}$ for $0 \leq v \leq N-1$, where the sum is performed modulo $2 \mathrm{~N}$.

In the context of this paper, we recall some properties of these graphs

Lemma 2.1 ([1]). Let $N \geq 5$ be an integer, and let $k_{1}, \ldots, k_{m}$ be a sequence of different odd elements from $\mathbb{Z}_{2 N}$. The following statements hold:

(i) The graph $S_{2 N}\left(k_{1}, \ldots, k_{m}\right)$ is m-regular, bipartite and has at most $m$ Cayley colors in $\mathbb{Z}_{2 N}$. Moreover, the girth of $S_{2 N}\left(k_{1}, \ldots, k_{m}\right)$ is at least 6 iff all the numbers $k_{i}-k_{j}$ are different in $\mathbb{Z}_{2 N}$, for $i \neq j$ and $1 \leq i, j \leq m$.

(ii) Let $0<P, Q<N$ be two different even elements from $\mathbb{Z}_{2 N}$. The graph $S_{2 N}\left(P, Q ; k_{1}, \ldots, k_{m}\right)$ is $(m+2)$-regular and has at most $m+2$ Cayley colors. Moreover, the girth of $S_{2 N}\left(P, Q ; k_{1}, \ldots, k_{m}\right)$ is at least 5 iff the following conditions hold in $\mathbb{Z}_{2 N}$ :

(a) The numbers $3 P, 4 P, 3 Q, 4 Q$ are different from 0.

(b) All the numbers $k_{i}-k_{j}$ are different for $i \neq j$ and $1 \leq i, j \leq m$.

(c) $k_{i}-k_{j} \neq a-a^{\prime}$ for every pair $a, a^{\prime} \in\{0, \pm P, \pm Q\}$.

Lemma $2.2([1])$. Let $k_{1}, \ldots, k_{m}$ be a sequence of different odd integers such that all the numbers $k_{i}-k_{j}$ are different in $\mathbb{Z}$, for $i \neq j$ and $1 \leq i, j \leq m$; and let $P, Q$ be two positive even integers such that $k_{i}-k_{j} \neq a-a^{\prime}$ for every pair $a, a^{\prime} \in\{0, \pm P, \pm Q\}$.

The following statements hold:

(i) For every integer $N>\max \left\{\left|k_{i}\right|, k_{i}-k_{j}\right\}$, the graph $S_{2 N}\left(k_{1}, \ldots, k_{m}\right)$ is m-regular, bipartite with girth at least 6 and has at most $m$ Cayley colors in $\mathbb{Z}_{2 N}$.

(ii) For every integer $N>\max \left\{\left|k_{i}\right|, k_{i}-k_{j}, a-a^{\prime}\right\}$ where $a, a^{\prime} \in\{0, \pm P, \pm Q\}$, the graph $S_{2 N}\left(P, Q ; k_{1}, \ldots, k_{m}\right)$ is $(m+2)$ regular, with girth at least 5 and has at most $m+2$ Cayley colors.

The graphs described in Definition 2.6 are used in the rest of this paper. They will be given either by explicit constructions, or by using their relationship with finite difference sets.

\section{Results for small values, $11 \leq q \leq 29$}

We provide suitable graphs $\left(G_{L}, G_{P}, H_{L}, H_{P}\right)$ with $V\left(G_{L}\right), V\left(G_{P}\right), V\left(H_{L}\right), V\left(H_{P}\right) \subseteq \mathbb{Z}_{q-1}$ to construct $\mathcal{L}_{q}\left(G_{L}, G_{P}, H_{L}, H_{P}\right)$ and improve $\operatorname{rec}(k, 5)$ for $k \in\{13,14,21,23,25,27,29,31,33\}$. Applying Remark 2.1 we also obtain new values of $r e c(k, 5)$ for $k \in\{24,26,28,30,32\}$.

Theorem 3.1. The upper bounds on $n(k, 5)$ listed in the last column of Table 1 for $k \in\{13,14,21,23,24, \ldots, 33\}$ provide better values of $\operatorname{rec}(k, 5)$.

Proof. For $q \in\{11,13,19,23,25,27,29\}$, let us consider the graph $\mathcal{L}_{q}$ given in Definition 2.1. Next we provide graphs $\left(G_{L}, G_{P}, H_{L}, H_{P}\right), r$-suitable for amalgamation into $\mathcal{L}_{q}$ with $r=k-q$. Theorem 2.2 guarantees that the graph $\mathcal{L}_{q}\left(G_{L}, G_{P}, H_{L}, H_{P}\right)$ is $k$-regular and has girth at least 5 .

$\rightarrow q=11 \rightarrow k=13$ :

Consider $q=11, r=2, W_{L}=W_{P}=\emptyset, T_{L}=\mathbb{Z}_{10}$ and $T_{P}=\{0,4,5,9\}$. Let $G_{L}$ be a Petersen graph with $V\left(G_{L}\right)=\mathbb{Z}_{10}$, two disjoint pentagons $(0,2,4,6,8,0),(1,5,9,3,7,1)$ and edges $\{0,1\},\{2,3\},\{4,5\},\{6,7\},\{8,9\}$ joining them. Hence, the set of Cayley colors of $G_{L}$ (in $\mathbb{Z}_{10}$ ) is $\omega\left(G_{L}\right)= \pm\{1,2,4\}$. Let $G_{P}$ be with $V\left(G_{P}\right)=\mathbb{Z}_{10}$ formed by $S_{10}(3,-3)$ and the chords $\{0,5\},\{4,9\}$. The set of Cayley colors of $G_{P}$ (in $\left.\mathbb{Z}_{10}\right)$ is $\omega\left(G_{P}\right)= \pm\{3,5\}$, and clearly, $\omega\left(G_{L}\right) \cap \omega\left(G_{P}\right)=\emptyset$. By Theorem 2.2, $H_{P}=\emptyset$ and the vertices of $H_{L}$ are the vertices of degree 2 in $G_{P}$, that is, $V\left(H_{L}\right)=\{1,2,3,6,7,8\}$. Let $H_{L}$ be the 6-cycle $(1,2,3,8,7,6,1)$ which satisfies the condition $E\left(H_{L}\right) \cap E\left(G_{P}\right)=\emptyset$. Since $\left(G_{L}, G_{P}, H_{L}, H_{P}\right)$ is an ordered set of $r$-suitable graphs, the amalgam $\mathcal{L}_{11}\left(G_{L}, G_{P}, H_{L}, \emptyset\right)$ is 13-regular, has girth 5 and $2\left(11^{2}-1\right)-10-4=226$ vertices. It states $\operatorname{rec}(13,5)=226$.

$\neg q=13 \rightarrow k=14$ :

Consider $q=13, W_{L}=\{2,7\}, W_{P}=\{7,11\}, T_{L}=T_{P}=\emptyset$ and $r=1$. Let $G_{L}$ be a Petersen graph formed by the disjoint pentagons $(1,6,11,4,9,1),(0,5,10,3,8,0)$ and edges $\{1,8\},\{6,5\},\{11,3\},\{4,0\},\{9,10\}$ joining them. The set of Cayley colors of $G_{L}$ (in $\mathbb{Z}_{12}$ ) is $\omega\left(G_{L}\right)= \pm\{1,4,5\}$. Let $G_{P}$ be a Petersen graph with disjoint pentagons $(0,9,6,8,10,0)$, $(2,5,3,1,4,2)$, and edges $\{0,2\},\{9,3\},\{6,4\},\{8,5\},\{10,1\}$ joining them. The set of Cayley colors of $G_{P}\left(\right.$ in $\left.\mathbb{Z}_{12}\right)$ is $\omega\left(G_{P}\right)=$ $\pm\{2,3,6\}$, so $\omega\left(G_{L}\right) \cap \omega\left(G_{P}\right)=\emptyset$. By Theorem $2.2, V\left(H_{L}\right)=\mathbb{Z}_{12} \backslash W_{P}, V\left(H_{P}\right)=\mathbb{Z}_{12} \backslash W_{L}$ and the degree of both $H_{L}$ and $H_{P}$ must be equal to $r=1$. Let $H_{L}$ be formed by the edges $\{0,1\},\{2,3\},\{4,5\},\{8,9\},\{6,10\}$ and let $H_{P}$ be formed by the edges $\{0,3\},\{1,4\},\{6,9\},\{8,10\},\{5,11\}$. Notice that $E\left(H_{P}\right) \cap E\left(G_{L}\right)=\emptyset$ and $E\left(H_{L}\right) \cap E\left(G_{P}\right)=\emptyset$. Since the ordered set $\left(G_{L}, G_{P}, H_{L}, H_{P}\right)$ is $r$-suitable, the graph $\mathcal{L}_{13}\left(G_{L}, G_{P}, H_{L}, H_{P}\right)$ is 14 -regular, has girth 5 and order $2\left(13^{2}-1\right)-56=280$. This provides rec $(14,5)=280$. 
$\checkmark q=19 \rightarrow k=21:$

For $q=19$, consider $r=2, W_{L}=W_{P}=\{6\}, T_{L}=\{0,2,4,5,7,8,10,13,14,15,16\}$ and $T_{P}=\{0,1,3$, $4,5,8,11,12,13,16,17\}$. Let $G_{L}$ be the $(3,4)$-regular graph formed by the hamiltonian cycle $(0,5,12,13,2,16,3$, $8,7,11,15,1,14,10,9,4,17,0)$ and the paths $(1,0,7,2,9),(3,14,13,17),(11,10,5,16,15,4,8,12)$. Let $G_{P}$ be the $(3,4)$-regular graph formed by the hamiltonian cycle $(0,9,11,13,10,8,2,12,4,7,17,1,16,14,5,3,15,0)$ and the paths $(2,17,11,14),(9,12,3,1,10),(7,5,8,0,16,4,13,15)$. Since $\omega\left(G_{L}\right)= \pm\{1,4,5,7\}$ and $\omega\left(G_{P}\right)= \pm\{2,3,6,8,9\}$ in $\mathbb{Z}_{18}$, we have $\omega\left(G_{L}\right) \cap \omega\left(G_{P}\right)=\emptyset$. Graphs $H_{L}$ and $H_{P}$ with $V\left(H_{L}\right)=\mathbb{Z}_{18} \backslash\left(W_{P} \cup T_{P}\right)$ and $V\left(H_{P}\right)=\mathbb{Z}_{18} \backslash\left(W_{L} \cup T_{L}\right)$ are formed by the cycles $(2,7,14,15,10,9,2)$ and $(1,3,9,11,17,12,1)$, respectively. Notice that $E\left(H_{L}\right) \cap E\left(G_{P}\right)=E\left(H_{P}\right) \cap E\left(G_{L}\right)=\emptyset$. Since all the requirements of suitability are satisfied, the graph $\mathcal{L}_{17}\left(G_{L}, G_{P}, H_{L}, H_{P}\right)$ has degree 21 and girth 5 . Hence $\operatorname{rec}(21,5)=2\left(19^{2}-1\right)-40-22=658$.

$\checkmark q=23 \rightarrow k=23:$

Consider $q=23, r=0, W_{L}=\{5,10,20\}, W_{P}=\{1,10,17\}, T_{L}=\mathbb{Z}_{22} \backslash W_{L}$ and $T_{P}=\mathbb{Z}_{22} \backslash W_{P}$. Since $G_{L}$ and $G_{P}$ must have degree 4 and girth 5 , both must be isomorphic to the unique $(4,5)$-cage $[14,16]$. Consider the graph $G_{L}$ with vertex set $V\left(G_{L}\right)=T_{L}$ formed by the two hamiltonian cycles $(0,18,4,11,7,3,8,9,13,12,19,15,14,21,16,1,6,2,17,0)$, $(0,7,12,16,17,9,4,21,3,2,19,18,1,8,15,11,6,13,14,0)$ and the graph $G_{P}$ with $V\left(G_{P}\right)=T_{P}$ formed by the two cycles $(0,16,13,2,12,21,5,7,4,6,3,14,20,11,8,18,15,9,19,0),(0,12,6,8,19,7,13,11,21,9,3,16,18,5,14,2,15$, 4, 20, 0). According to Definition $2.5(i i), V\left(H_{L}\right)=V\left(H_{P}\right)=\emptyset$. Since $\omega\left(G_{L}\right)= \pm\{1,4,5,7,8\}$ and $\omega\left(G_{P}\right)= \pm\{2,3,6,9,10,11\}$ in $\mathbb{Z}_{22}$, the suitability condition $\omega\left(G_{L}\right) \cap \omega\left(G_{P}\right)=\emptyset$ is satisfied and the graph $\mathcal{L}_{23}\left(G_{L}, G_{P}, \emptyset, \emptyset\right)$ has degree 23 and girth 5 with $2\left(23^{2}-1\right)-24 \cdot 6-38=874$ vertices. Then, $\operatorname{rec}(23,5)=874$.

$\checkmark q=23 \rightarrow k=25,24:$

To construct a $(25,5)$-regular graph, consider $q=23, r=2, T_{L}=T_{P}=\emptyset, W_{L}=\{2,7\}$ and $W_{P}=\{7,14\}$. Let $G_{L}$ be the 4-regular graph formed by the cycles $(4,11,6,13,12,19,4),(0,17,21,3,8,1,18,14,10,9,16,15,20,5,0)$, $(0,4,9,1,6,5,10,3,11,15,14,13,21,20,19,18,17,16,12,8,0)$, and let $G_{P}$ be the 4-regular graph formed by the cycles $(0,9,6,4,13,16,18,12,15,17,20,11,21,19,3,1,10,8,5,2,0),(9,15,13,11,5,3,9),(0,10,21,12,2,4,1,20,18,6$, $8,17,19,16,0)$. Since in $\mathbb{Z}_{22}, \omega\left(G_{L}\right)= \pm\{1,4,5,7,8\}$ and $\omega\left(G_{P}\right)= \pm\{2,3,6,9,10,11\}$, we have $\omega\left(G_{L}\right) \cap \omega\left(G_{P}\right)=\emptyset$. Let $H_{L}$ and $H_{P}$ be the cycles $(0,4,3,8,12,16,20,2,6,10,18,11,15,19,1,5,13,9,17,21,0)$ and $(0,3,6,9,12,15,18,5,21$, $1,4,10,13,16,19,8,11,14,17,20,0)$, respectively. Clearly, $V\left(H_{L}\right)=\mathbb{Z}_{22} \backslash W_{P}, V\left(H_{P}\right)=\mathbb{Z}_{22} \backslash W_{L}$. Also, $E\left(H_{L}\right) \cap E\left(G_{P}\right)=\emptyset$ and $E\left(H_{P}\right) \cap E\left(G_{L}\right)=\emptyset$. Since the suitability conditions are satisfied, the graph $\mathcal{L}_{23}\left(G_{L}, G_{P}, H_{L}, H_{P}\right)$ has girth 5, is 25 -regular and has $2\left(23^{2}-1\right)-4 \cdot 24=960$ vertices which gives the new value $\operatorname{rec}(25,5)=960$. Moreover, from Remark $2.1(3)$ we have the new bound $\operatorname{rec}(24,5)=920$.

$\checkmark q=23 \rightarrow k=27,26$ :

To construct a $(27,5)$-regular graph, consider $q=23, r=4, W_{L}=W_{P}=T_{L}=\varnothing$ and $T_{P}=\{6,17\}$. Let $G_{L}=S_{22}(4,8 ; 1,-1)$, which is a 4-regular graph of girth 5 with $\omega\left(G_{L}\right)= \pm\{1,4,8\}$. Let $G_{P}$ be formed by the cycle $S_{22}(7,-7)$ together with the three paths $(6,16,3,20,8,21,11,17),(6,12,18,15,2,13,4,7,5,17)$ and $(6,0,10,19,9,14,5,17)$. Hence, $G_{P}$ is a (4,5)-regular graph of girth 5 with $\omega\left(G_{P}\right)= \pm\{3,5,6,7,9,10,11\}$. Clearly $\omega\left(G_{L}\right) \cap \omega\left(G_{P}\right)=\emptyset$. Let $H_{L}$ be the graph with $V\left(H_{L}\right)=\mathbb{Z}_{22} \backslash T_{P}$ formed by the cycles $(0,9,8,4,5,11,3,2,20,16,12,13,14,10,1,21,15,7,18,19,0)$, $(0,13,5,18,10,9,11,16,15,14,3,19,21,4,20,0),(1,2,7,8,12,1)$ and $H_{P}=S_{22}(6,10 ; 7,-7)$. It is easy to check that $H_{L}$ and $H_{P}$ are 4-regular graphs of girth 5 such that $E\left(H_{L}\right) \cap E\left(G_{P}\right)=\emptyset, E\left(H_{P}\right) \cap E\left(G_{L}\right)=\emptyset$. By Theorem $2.2, \mathcal{L}_{23}\left(G_{L}, G_{P}, H_{L}, H_{P}\right)$ has girth 5 , is 27 -regular and has $2\left(23^{2}-1\right)-2=1054$ vertices. This provides $\operatorname{rec}(27,5)=1054$. Remark $2.1(2)$ states $\operatorname{rec}(26,5)=1010$.

$\checkmark q=25 \rightarrow k=29,28$ :

For $q=25$, consider $r=4, W_{L}=W_{P}=\emptyset$ and $T_{L}=T_{P}=\{7,11,19,23\}$. Let $G_{L}$ be the graph $S_{24}(2,10 ; 3,-3)$ together with the edges $\{7,19\},\{11,23\}$. Let $G_{P}$ be formed by the cycles $(2,17,18,11,10,14,20,19,2),(1,5,6,22,3,9,8,21,1)$, $(0,4,23,16,12,13,7,15,0),(0,1,17,23,8,19,12,3,14,7,6,11,0),(2,15,9,18,13,21,10,16,5,20,4,22,2)$, and the edges $\{7,23\},\{11,19\}$. Let $H_{L}$ be formed by the cycles $(6,13,5,12,10,9,17,16,15,4,21,2,3,8,0),(0,16,20,13,4,10$, $1,3,15,14,12,2,6,9,22,8,5,17,21,18,0),(1,18,14,22,20)$, and $H_{P}$ by the cycles $(0,10,14,20,9,3,4,5,1,8,17,2$, 13, 21, 15, 0), (0,12, 8, 14, 13, 18, 3, 10, 22, 2, 9, 1, 21, 16, 4, 17, 15, 20, 6, 5, 0) and (18, 6, 22, 16, 12). The graphs $G_{L}, G_{P}$ $H_{L}, H_{P}$ have girth $5, E\left(H_{L}\right) \cap E\left(G_{P}\right)=\emptyset, E\left(H_{P}\right) \cap E\left(G_{L}\right)=\emptyset$ and $\omega\left(G_{L}\right) \cap \omega\left(G_{P}\right)=\varnothing$ because $\omega\left(G_{L}\right)= \pm\{2,3,10,12\}$ and $\omega\left(G_{P}\right)= \pm\{1,4,5,6,7,8,9,11\}$. Since the ordered set $\left(G_{L}, G_{P}, H_{L}, H_{P}\right)$ is $r$-suitable, the graph $\mathcal{L}_{25}\left(G_{L}, G_{P}, H_{L}, H_{P}\right)$ is 29-regular, has girth 5 and order $2\left(25^{2}-1\right)-8=1240$, proving that $\operatorname{rec}(29,5)=1240$. Moreover, Remark 2.1 (2) states $\operatorname{rec}(28,5)=1192$.

$\checkmark q=27 \rightarrow k=31,30:$

To construct a (31, 5)-regular graph, consider $q=27, r=4, W_{L}=W_{P}=\varnothing$ and $T_{L}=T_{P}=\{5,7,9,19,21,23\}$. Let $G_{L}$ be the (4, 5)-regular graph with $V\left(G_{L}\right)=\mathbb{Z}_{26}$ and $E\left(G_{L}\right)=E\left(S_{26}(4,8,1,-1)\right) \cup\{\{5,19\},\{7,21\},\{9,23\}\}$, and let $G_{P}$ be formed by the cycles $(0,10,3,16,22,13,4,11,14,12,1,17,24,0),(0,2,11,8,10,5,14,9,3,23,12,18,16,25,1,6,22$, $20,17,7,13,19,24,21,4,15,0),(2,23,20,15,9,6,21,5,25,19,8,18,7,2)$, and the edges $\{5,7\},\{9,19\},\{21,23\}$. Since $\omega\left(G_{L}\right)= \pm\{1,4,8,12\}$ and $\omega\left(G_{P}\right)= \pm\{2,3,5,6,7,9,10,11,13\}$ in $\mathbb{Z}_{26}$, it follows that $\omega\left(G_{L}\right) \cap \omega\left(G_{P}\right)=\emptyset$. Let $H_{L}$ be the 4-regular graph with $V\left(H_{L}\right)=\mathbb{Z}_{26} \backslash T_{P}$ and cycles $(0,16,12,13,17,10,11,3,15,22,14,2,1,24,8,4,20,6,18,25,0)$, $(0,1,13,20,14,10,24,6,16,15,17,18,2,3,4,0),(11,25,22,8,12,11)$, and $H_{P}$ be the 4-regular graph with $V\left(H_{P}\right)=$ $\mathbb{Z}_{26} \backslash T_{L}$ with cycles $(0,11,8,25,13,18,3,10,22,2,12,17,24,16,1,20,15,6$,

$4,14,0),(0,10,25,6,12,3,1,14,17,8,20,2,13,24,15,0),(18,4,22,16,11,18)$. Since all the suitability conditions are satisfied, bounds (1) and (2) in Remark 2.1 establish $\operatorname{rec}(31,5)=1444$ and $\operatorname{rec}(30,5)=1392$, respectively. 
$\checkmark=29 \rightarrow k=33,32:$

Consider $q=29, r=4, T_{L}=T_{P}=\{2,4,7,11,16,18,21,25\}$ and $W_{L}=W_{P}=\varnothing$. Let $G_{L}$ be the graph with $E\left(G_{L}\right)=E\left(S_{28}(4,8 ; 1,-1)\right) \cup\{\{2,16\},\{4,18\},\{7,21\},\{11,25\}\}$ and let $G_{P}$ be the graph formed by the hamiltonian cycle $(0,22,12,2,20,9,11,6,8,24,15,17,14,21,27,10,3,16,19,25,7,5,26,23,4,1,13,18,0)$, the 28-length-path $(1,22,9,3,5,18,8,25,10,13,11,23,2,14,19,26,24,21,0,16,6,17,7,20,27,4,15,12)$ and the edges $\{1,7\},\{2,18\}$, $\{4,16\},\{11,21\},\{12,25\}$. Since $\omega\left(G_{L}\right)= \pm\{1,4,8,14\}$ and $\omega\left(G_{P}\right)= \pm\{2,3,5,6,7,9,10,11,12,13\}$ in $\mathbb{Z}_{28}$, it follows that $\omega\left(G_{L}\right) \cap \omega\left(G_{P}\right)=\varnothing$. Consider the 4-regular graph $H_{L}$ with cycles $(0,23,12,20,14,10,8,3,15,27,6,26,1,9,13,24,5,17$, $22,19,0),(8,19,27,13,12,8),(0,1,20,5,6,10,9,17,23,15,14,22,26,3,24,0)$; and the isomorphic graph $H_{P}$ formed by the cycles $(3,17,20,22,12,3),(0,10,23,26,8,1,27,9,5,14,24,6,13,19,15,0),(0,3,14,23,13,17,1,10,22,6,8$, $5,19,12,27,24,15,26,20,9,0)$. The graphs $G_{L}, G_{P}, H_{L}$ and $H_{P}$ are $r$-suitable, and bounds $(1)$ and $(2) \operatorname{state} r e c(33,5)=1664$ and $\operatorname{rec}(32,5)=1608$, respectively.

\section{Results for greater values, $61 \leq q \leq 479$}

In the section above, new values of $\operatorname{rec}(k, 5)$ are obtained by amalgams into $\mathcal{L}_{q}$, for prime powers $11 \leq q \leq 29$. In the next theorem we focus on $q=61$ and $q \geq 71$ and amalgamate regular graphs $G_{L}$, $G_{P}$ with degrees $r_{q}>6$; similarly as in [9] for $r_{q}=4$ and as in [1] for $r_{q}=5,6$.

Theorem 4.1. Let $q \geq 61$ be an odd prime power. Then $n\left(q+r_{q}, 5\right) \leq 2\left(q^{2}-1\right)$ where $r_{q}$ is the following integer:

$$
r_{q}=\left\{\begin{array}{cl}
7 & \text { if } q \in\{61,71, \ldots, 89\} \\
8 & \text { if } q \in\{101, \ldots, 109\} \\
9 & \text { if } q \in\{97,113, \ldots, 139\}-\{127,131\} \\
10 & \text { if } q \in\{127,131,149, \ldots, 181\} \\
11 & \text { if } q \in\{191, \ldots, 223\} \\
12 & \text { if } q \geq 227 \text { and } q \neq 241 \\
13 & \text { if } q=241
\end{array}\right.
$$

Proof. For each odd prime power $q \geq 61$ (except $q=67$ ) we construct a definite pair of suitable $r_{q}$-regular graphs $G_{L}$ and $G_{P}$. Theorem 2.1 proves that the amalgam $\mathcal{L}_{q}\left(G_{L}, G_{P}\right)$ is $\left(q+r_{q}\right)$-regular with girth at least 5 and order $2\left(q^{2}-1\right)$. Next, we provide these two graphs for $q \in\{61,97,127,131\}$ and $q \geq 263$. For the rest of values $q$ the graphs $G_{L}, G_{P}$ are indicated in Table 2.

$\checkmark q=61 \rightarrow k=68:$

We consider the 7-regular graph $G_{L}=S_{60}(12,24 ; 1,-1,5,19,27)$. To construct another 7-regular graph $G_{P}$, we add to the 5-regular bipartite graph $S_{60}(3,7,15,-29,29)$ the edges $\{\{2 i, 2 i+10\}: 0 \leq i \leq 29\}$, and the edges of the five cycles $(1+2 i, 31+2 i, 11+2 i, 51+2 i, 21+2 i, 41+2 i, 1+2 i)$, for $0 \leq i \leq 4$. Since both $G_{L}$ and $G_{P}$ have girth 5 and $\omega\left(G_{L}\right) \cap \omega\left(G_{P}\right)=\varnothing$, due to $\omega\left(G_{L}\right)= \pm\{12,24,1,5,19,27\}$ and $\omega\left(G_{P}\right)= \pm\{10,20,30,3,7,15,29\}$, the graph $\mathcal{L}_{61}\left(G_{L}, G_{P}\right)$ yields $n(61+7,5) \leq 2\left(61^{2}-1\right)=7440$.

$\rightarrow q=97 \rightarrow k=106:$

Let $G_{L}$ be the graph with $V\left(G_{L}\right)=\mathbb{Z}_{96}$ and edges the ones in $S_{96}(1,-1,7,19,-23,-27,-37)$ (isomorphic to $\left.\mathcal{L}_{7}\right)$ together with the edges $\{2 i, 2 i+16\}$, for $0 \leq i \leq 47$, and the ones in the cycles $(1+2 i, 49+2 i, 17+2 i, 81+2 i, 33+2 i, 65+2 i, 1+2 i)$ , for $0 \leq i \leq 7$. The graph $G_{L}$ is 9 -regular, has girth 5 and set of Cayley colors $\omega\left(G_{L}\right)= \pm\{16,32,48,1,7,19,23,27,37\}$. Let $G_{P}$ be the isomorphic graph obtained from $G_{L}$ by the permutation on $V\left(G_{L}\right)=\mathbb{Z}_{96}$ expressed as the following product of cycles:

(12962 37199055777646271811842$) \cdot(3483017396368422566416440241473823677043724426$ $1528161056986538984523220126) \cdot(98392563421795835825188543360362213) \cdot(31718778477374$ $45939585618050) \cdot(4959) \cdot(81919457)$.

The set $\omega\left(G_{P}\right)=\mathbb{Z}_{96} \backslash\left(\{0\} \cup \omega\left(G_{L}\right)\right)$, and the graph $\mathcal{L}_{96}\left(G_{L}, G_{P}\right)$ yields $n(96+9,5) \leq 2\left(97^{2}-1\right)$.

$\checkmark q=127 \rightarrow k=137$ :

We start with the graph $S_{126}(1,-1,5,13,29,39,-45,61)$ which is isomorphic to $\mathcal{L}_{8}$, and consider the graph $G_{L}=$ $S_{126}(18,36 ; 1,-1,5,13,29,39,-45,61)$. It is 10 regular, has girth 5 and $\omega\left(G_{L}\right)= \pm\{18,36,1,5,13,29,39,45,61\}$. The isomorphic graph $G_{P}$ is obtained from $G_{L}$ by the following permutation on $V\left(G_{L}\right)=\mathbb{Z}_{126}$ :

(1 10678592312381273955115442319679671171211151263103531128663991051027658443221 419826194725643124927111688644737523628191096980604512598745751108846246336525118 $891051148710477107122911310075101110859397112618147120907050356648342216842) \cdot(1538$ $29403020) \cdot(17684924) \cdot(113119)$.

Since $\omega\left(G_{P}\right) \cap \omega\left(G_{L}\right)=\emptyset$, the graph $\mathcal{L}_{127}\left(G_{L}, G_{P}\right)$ establishes $n(127+10,5) \leq 2\left(127^{2}-1\right)$.

- $q=131 \rightarrow k=141:$

Consider the 10-regular graph $G_{L}=S_{130}(26,52 ; 1,-1,7,-13,-31,-35,55,-59)$ and the isomorphic graph $G_{P}$ obtained by the following permutation on $V\left(G_{L}\right)=\mathbb{Z}_{130}$ :

(1) 34241263110837977765240273511199118871211269195544156422821584339117103123122 89115102725032233318938262010513737451171168664463022119468483137362514711912088 
Table 2

The $r_{q}$-regular graphs $G_{L}$ and $G_{P}$ are suitable for amalgamation into $\mathcal{L}_{q}$.

\begin{tabular}{|c|c|c|c|}
\hline$q$ & $r_{q}$ & $G_{L}$ & $G_{P}$ \\
\hline 61 & 7 & $S_{60}(12,24 ; 1,-1,5,19,27)$ & See proof of Theorem 4.1 \\
\hline 71 & 7 & $S_{70}(10,20 ; 1,-1,5,13,-23)$ & $S_{70}(2,4 ; 3,-7,15,-21,31)$ \\
\hline 73 & 7 & $S_{72}(16,28 ; 1,-1,5,-9,25)$ & $S_{72}(2,4 ; 3,-7,15,-23,35)$ \\
\hline 79 & 7 & $S_{78}(12,24 ; 1,-1,5,-9,-27)$ & $S_{78}(2,4 ; 3,-7,15,-21,31)$ \\
\hline 81 & 7 & $S_{80}(12,24 ; 1,-1,5,-9,-29)$ & $S_{80}(2,4 ; 3,-7,15,-21,-39)$ \\
\hline 83 & 7 & $S_{82}(12,24 ; 1,-1,5,-9,-27)$ & $S_{82}(2,4 ; 3,-7,15,-21,31)$ \\
\hline 89 & 7 & $S_{88}(12,24 ; 1,-1,5,-9,-27)$ & $S_{88}(2,4 ; 3,-7,15,-21,33)$ \\
\hline 97 & 9 & See proof of Theorem 4.1 & See proof of Theorem 4.1 \\
\hline 101 & 8 & $S_{100}(16,32 ; 1,-1,5,-9,-21,-39)$ & $S_{100}(2,4 ; 3,-7,15,-23,33,-43)$ \\
\hline 103 & 8 & $S_{102}(16,32 ; 1,-1,5,-9,-21,35)$ & $S_{102}(2,4 ; 3,-7,15,-23,-43,45)$ \\
\hline 107 & 8 & $S_{106}(16,32 ; 1,-1,5,-9,-21,29)$ & $S_{106}(2,4 ; 3,-7,15,-23,33,47)$ \\
\hline 109 & 8 & $S_{108}(16,32 ; 1,-1,5,-9,-21,29)$ & $S_{108}(2,4 ; 3,-7,15,-23,33,-43)$ \\
\hline 113 & 9 & $S_{112}(18,36 ; 1,-1,27,31,43,-47,51)$ & $S_{112}(2,4 ; 3,-15,25,-29,37,-39,53)$ \\
\hline 121 & 9 & $S_{120}(18,36 ; 1,-1,5,-11,-25,-33,45)$ & $S_{120}(6,12 ; 3,-7,-29,-37,41,55,57)$ \\
\hline 125 & 9 & $S_{124}(18,36 ; 1,-1,5,15,-25,-33,-45)$ & $S_{124}(2,4 ; 3,-7,-19,21,-39,47,-53)$ \\
\hline 127 & 10 & $S_{126}(18,36 ; 1,-1,5,13,29,39,-45,61)$ & See proof of Theorem 4.1 \\
\hline 131 & 10 & $S_{130}(26,52 ; 1,-1,7,-13,-31,-35,55,-59)$ & See proof of Theorem 4.1 \\
\hline 137 & 9 & $S_{136}(18,36 ; 1,-1,5,-9,21,49,61)$ & $S_{136}(2,4 ; 3,-7,15,-23,33,-43,65)$ \\
\hline 139 & 9 & $S_{138}(18,36 ; 1,-1,5,-9,21,33,-47)$ & $S_{138}(2,4 ; 3,-7,15,-23,35,49,-59)$ \\
\hline 149 & 10 & $S_{148}(18,36 ; 1,-1,7,-21,31,41,45,57)$ & $S_{148}(2,4 ; 3,-9,17,-25,-43,53,-63,-73)$ \\
\hline 151 & 10 & $S_{150}(18,36 ; 1,-1,5,15,-23,45,57,65)$ & $S_{150}(2,4 ; 3,-7,19,-21,37,49,-59,69)$ \\
\hline 157 & 10 & $S_{156}(18,36 ; 1,-1,5,13,-29,-39,-61,69)$ & $S_{156}(2,4 ; 3,-7,19,-21,-43,-55,63,-73)$ \\
\hline 163 & 10 & $S_{162}(18,36 ; 1,-1,5,-9,-25,-47,-59,75)$ & $S_{162}(2,4 ; 3,-7,15,-21,31,-41,-71,73)$ \\
\hline 167 & 10 & $S_{166}(18,36 ; 1,-1,5,-9,21,-41,49,-65)$ & $S_{166}(2,4 ; 3,-7,15,-23,33,-55,69,-69)$ \\
\hline 169 & 10 & $S_{168}(18,36 ; 1,-1,5,-9,-21,47,-59,81)$ & $S_{168}(2,4 ; 3,-7,15,-23,43,-53,67,-67)$ \\
\hline 173 & 10 & $S_{172}(18,36 ; 1,-1,5,-9,21,49,61,-77)$ & $S_{172}(2,4 ; 3,-7,15,-23,39,57,-67,-81)$ \\
\hline 179 & 10 & $S_{178}(18,36 ; 1,-1,5,-9,21,33,-61,79)$ & $S_{178}(2,4 ; 3,-7,15,-23,35,-59,-73,75)$ \\
\hline 181 & 10 & $S_{180}(18,36 ; 1,-1,5,-9,21,33,-61,79)$ & $S_{180}(2,4 ; 3,-7,15,-23,35,-37,59,-85)$ \\
\hline 191 & 11 & $S_{190}(18,36 ; 1,-1,5,15,-25,43,63,75,-81)$ & $S_{190}(2,4 ; 3,-7,17,-23,-41,49,-61,79,-89)$ \\
\hline 193 & 11 & $S_{192}(18,36 ; 1,-1,5,13,29,39,-45,61,81)$ & $S_{192}(4,8 ; 3,-3,17,-25,-35,43,73,-83,-85)$ \\
\hline 197 & 11 & $S_{196}(18,36 ; 1,-1,5,-9,-33,49,-61,79,95)$ & $S_{196}(2,4 ; 3,-7,15,35,-41,51,65,-67,89)$ \\
\hline 199 & 11 & $S_{198}(18,36 ; 1,-1,5,-9,-21,39,-51,67,83)$ & $S_{198}(2,4 ; 3,-7,15,-23,-43,55,69,87,-87)$ \\
\hline 211 & 11 & $S_{210}(18,36 ; 1,-1,5,-9,21,33,-57,85,-101)$ & $S_{210}(2,4 ; 3,-7,15,-23,-37,47,-71,-95,97)$ \\
\hline 223 & 11 & $S_{222}(18,36 ; 1,-1,5,-9,21,33,-47,91,-107)$ & $S_{222}(2,4 ; 3,-7,15,-23,35,49,-51,-75,97)$ \\
\hline 227 & 12 & $S_{226}(18,36 ; 1,-1,5,-9,33,49,-65,79,-85,101)$ & $S_{226}(2,4 ; 3,-7,15,35,-37,51,69,-93,95,-107)$ \\
\hline 229 & 12 & $S_{228}(12,30 ; 1,-1,5,-9,-45,57,73,-79,95,-107)$ & $S_{228}(18,42 ; 3,-3,11,31,41,43,-61,93,97,-109)$ \\
\hline 233 & 12 & $S_{232}(36,72 ; 1,-1,5,25,-39,47,59,75,-95,-105)$ & $S_{232}(4,8 ; 3,-7,-35,43,57,63,-69,87,-87,89)$ \\
\hline 239 & 12 & $S_{238}(18,36 ; 1,-1,-5,11,-29,33,59,-85,103,111)$ & $S_{238}(2,4 ; 3,-7,19,-37,51,63,-71,87,-89,-109)$ \\
\hline 241 & 13 & $S_{240}(24,48 ; 1,-1,-5,9,27,39,-55,61,-75,-91,107)$ & $S_{240}(72,96 ;-3,15,-15,23,25,29,-37,79,-83,99,115)$ \\
\hline 243 & 12 & $S_{242}(18,36 ; 1,-1,-5,9,-21,43,75,-89,101,113)$ & $S_{242}(2,4 ; 3,-7,15,-23,33,-59,79,-91,103,-119)$ \\
\hline 251 & 12 & $S_{250}(18,36 ; 1,-1,5,-9,25,47,-59,87,99,115)$ & $S_{250}(2,4 ; 3,-7,15,-21,-39,43,63,-65,-95,-111)$ \\
\hline 257 & 12 & $S_{256}(18,36 ; 1,-1,5,-9,21,-43,61,87,99,-111)$ & $S_{256}(2,4 ; 3,-7,15,-23,33,-55,57,-69,-89,93)$ \\
\hline 263 & 12 & $S_{262}(28,56 ; 1,-1,-13,61,65,91,97,21,107,115)$ & $S_{262}(20,40 ; 3,-15,27,-47,49,55,57,-59,-63,-73)$ \\
\hline$\vdots$ & $\vdots$ & : & : \\
\hline$q$ & 12 & $S_{q-1}(28,56 ; 1,-1,-13,61,65,91,97,21,107,115)$ & $S_{q-1}(20,40 ; 3,-15,27,-47,49,55,57,-59,-63,-73)$ \\
\hline
\end{tabular}

$651271291251289267535519106817860442911284631148516842) \cdot(100719710882624515113101109$ $105124906647) \cdot(615910710480) \cdot(699870499396)$.

Since $\omega\left(G_{P}\right) \cap \omega\left(G_{L}\right)=\emptyset$, the graph $\mathcal{L}_{131}\left(G_{L}, G_{P}\right)$ establishes $n(131+10,5) \leq 2\left(131^{2}-1\right)$.

$>q \geq 263 \rightarrow k=q+12$ :

Consider the graphs $G_{L}=S_{q-1}(28,56 ; 1,-1,-13,61,65,91,97,21,107,115)$ and $G_{P}=S_{q-1}(20,40 ; 3,-15,27,-47$, $49,55,57,-59,-63,-73)$. The integers in their definitions verify the conditions in Lemma 2.2, so both of them are 12regular graphs with girth 5 when $q-1=2 N>260$, that is, when $q \geq 263$ is an odd prime power. As their Cayley colors are clearly disjoint, $G_{L}$ and $G_{P}$ form a suitable pair.

Let us notice that Theorem 4.1 is sharp when $q \in\{61,71,73,79,97,113,121,127,131,149$, $151,191,193,197,199\}$, because $\left|V\left(G_{L}\right)\right|=\left|V\left(G_{P}\right)\right|=q-1<\operatorname{rec}\left(r_{q}+1,5\right)$, so $r_{q}$ is the maximum degree that the chosen suitable graphs $G_{L}$ and $G_{P}$ can reach.

In [1] the authors consider some prime powers $31 \leq q \leq 59$ and $q=64,67$ to obtain bounds on $n(k, 5)$ for degrees $34 \leq k \leq 65$ and $69 \leq k \leq 73$. These values of $\operatorname{rec}(k, 5)$ are sharp and remain untouched. Now, we consider greater values of $k$. 
Corollary 4.1. Given an integer $k \in\{66,67,68\}$ and $k \geq 74$, let $q$ be the lowest odd prime power such that $k \leq q+r_{q}$, where $r_{q}$ is given in Table 2. Then, $n(k, 5) \leq 2\left(k-r_{q}+1\right)(q-1)$. In particular, $n(k, 5) \leq 2(k-11)(q-1)$ when $235 \leq k \leq q+12$.

Proof. Let $q \in\{61,71, \ldots$,$\} be an odd prime power, and let G_{L}, G_{P}$ be the suitable pair of graphs provided in the proof of Theorem 4.1. The graph $\mathcal{L}_{q}\left(G_{L}, G_{P}\right)$ is $\left(q+r_{q}\right)$-regular, has girth at least five and order $2(q+1)(q-1)$. Deleting iteratively from this graph $\gamma$ blocks of lines and $\gamma$ blocks of points (cf. Remark 2.1 (2)), we have $n\left(q+r_{q}-\gamma, 5\right) \leq 2(q+1-\gamma)(q-1)$, or equivalently, $n(k, 5) \leq 2\left(k-r_{q}+1\right)(q-1)$ for $k \leq q+r_{q}$.

In the following section we establish bounds better than the last one $n(k, 5) \leq 2(k-11)(q-1)$ for large odd prime powers $q$ and degrees $k$.

\section{Results for $q \in\{313,337,367\}$ and large values $q>479$}

In this section, thanks to a simple relationship between difference sets and graphs $S_{2 N}\left(k_{1}, \ldots, k_{m}\right)$, we construct three distinct families of graphs which are suitable for amalgamation into $\mathcal{L}_{q}$. This way, we obtain new bounds on $n(k, 5)$ for large values of $k$. In particular, we improve the known bound

$$
n\left(q+\left\lfloor\frac{\sqrt{q-1}}{4}\right\rfloor, 5\right) \leq 2\left(q^{2}-1\right) \text { for an odd prime power } q
$$

proved by Jørgensen (cf. [12], Corollary 19) using also difference sets. We begin with a simple auxiliar lemma.

Lemma 5.1. Let $m, N$ be two positive integers and $D=\left\{d_{1}, \ldots, d_{m}\right\} \subset \mathbb{Z}_{N}$ such that all the numbers $d_{i}-d_{j}$ with $i \neq j$ are different in $\mathbb{Z}_{N}$. Define $K=\left\{k_{1}, \ldots, k_{m}\right\}$ where $k_{i}=2 d_{i}+1 \in \mathbb{Z}_{2 N}$ and consider an even non-zero $\delta \in \mathbb{Z}_{2 N}$. Then:

(i) $S_{2 N}\left(k_{1}, \ldots, k_{m}\right)$ and $S_{2 N}\left(k_{1}-\delta, \ldots, k_{m}-\delta\right)$ are m-regular graphs with girth at least 6 .

(ii) $V=K \cap(K-\delta) \neq \emptyset$ iff $\delta=k_{i}-k_{j}$ for some $1 \leq i, j \leq m$. In this case, $V=\left\{k_{i}-\delta\right\}$.

(iii) $(-K) \cap(K-\delta) \neq \emptyset$ iff $\delta=k_{i}+k_{j}$ for some $1 \leq i, j \leq m$.

Proof. The assertion ( $i$ ) follows from Lemma 2.1. Items (ii) and (iii) follow from direct calculations. First, if $\alpha \in K \cap(K-\delta)$, there is an ordered pair $\left(k_{i}, k_{j}\right)$ with $\alpha=k_{j}=k_{i}-\delta$ and $\delta=k_{i}-k_{j}$ in $\mathbb{Z}_{2 N}$. Since $\left(k_{i}, k_{j}\right)$ is unique, we have $V=\left\{k_{i}-\delta\right\}$. In a similar way, if $\alpha \in(-K) \cap(K-\delta)$ then $\alpha=-k_{i}=k_{j}-\delta$ and $\delta=k_{i}+k_{j}$.

Roughly speaking, a subset $D \subset H$ of an group $H$ (written in additive notation) is a finite difference set if every non-zero element of $H$ can be represented by the same number $\lambda$ of differences $d-d^{\prime}$ with $d, d^{\prime} \in D$. We only need the first and simplest class of difference sets, that is, $\lambda=1$ and $H$ cyclic, defined and studied by Singer [17] in 1938.

Lemma 5.2 (cf. [17], Theorem 2). Given a prime power $p$, there is a subset $D=\left\{d_{0}, \ldots, d_{p}\right\} \subset \mathbb{Z}_{p^{2}+p+1}$ such that each non-zero element $h \in \mathbb{Z}_{p^{2}+p+1}$ is represented in a unique way as $h=d_{i}-d_{j}$.

We use these finite difference sets to obtain the following result:

Theorem 5.1. Let $p, q$ be prime powers such that $q=2\left(p^{2}+p+1\right)+1$. Then

$$
n\left(q+\frac{\sqrt{2 q-5}-1}{2}, 5\right) \leq 2 q^{2}-q-1 .
$$

Proof. Denote $N=p^{2}+p+1$ and let $\left\{d_{0}, \ldots, d_{p}\right\} \subset \mathbb{Z}_{N}$ be the difference set given by Lemma 5.2. Consider $K=$ $\left\{k_{0}, \ldots, k_{p}\right\} \subset \mathbb{Z}_{2 N}=\mathbb{Z}_{q-1}$ with $k_{i}=2 d_{i}+1$.

Since the set $B=\left\{k_{i}+k_{j}: 0 \leq i, j \leq p\right\}$ has only $(p+2)(p+1) / 2$ elements, take an even non-zero $\delta \in \mathbb{Z}_{2 N} \backslash B$ and its unique representation $\delta=k_{i_{\delta}}-k_{j_{\delta}}$. By Lemma 5.1 and the election of $\delta$, the $(p+1)$-regular graph $G_{L}=S_{q-1}\left(k_{0}, \ldots, k_{p}\right)$ and the $p$-regular graph $G_{P}=S_{q-1}\left(k_{0}-\delta, \ldots, k_{i_{\delta}-1}-\delta, k_{i_{\delta}+1}-\delta, \ldots, k_{p}-\delta\right)$ have girth al least 6 and disjoint sets of Cayley colors.

In order to apply Theorem 2.2 for $r=p, W_{L}=W_{P}=\emptyset, T_{L}=\mathbb{Z}_{q-1}$ and $T_{P}=\emptyset$, we define $H_{L}=S_{q-1}\left(k_{0}, \ldots, k_{p-1}\right)$ and $H_{P}=\emptyset$. Clearly, $E\left(H_{L}\right) \cap E\left(G_{P}\right)=\emptyset$ because $\omega\left(H_{L}\right) \cap \omega\left(G_{P}\right)=\emptyset$. The graph $\mathcal{L}_{q}\left(G_{L}, G_{P}, H_{L}, H_{P}\right)$ has girth at least 5 , order $2\left(q^{2}-1\right)-(q-1)=2 q^{2}-q-1$ and degree $q+p=q+\frac{1}{2}(\sqrt{2 q-5}-1)$.

Let us notice that the graph $S_{2\left(p^{2}+p+1\right)}\left(k_{0}, \ldots, k_{p}\right)$ is isomorphic to the incidence graph $\mathcal{P}_{p}$ of the projective plane. Actually, Singer proves the existence of finite difference sets as a consequence of a geometric property of the projective planes.

Now we turn our attention to difference sets of a group $H$ relative to a subgroup $H_{1}$. We are only interested in the differences defined by Bose [6] in 1942.

Lemma 5.3 (cf. [6], Theorem 1). Consider a prime power $p \geq 3$, the cyclic group $H=\mathbb{Z}_{p^{2}-1}$ and the subgroup $H_{1}=(p+1)$ formed by the multiples of $p+1$. Then there exists a set $D=\left\{d_{1}, \ldots, d_{p}\right\} \subset H$ such that each element $h \in H \backslash H_{1}$ is represented as $d_{i}-d_{j}$ in a unique way, and no difference $d_{i}-d_{j}$ with $i \neq j$ lies in $H_{1}$. 
Bose's relative difference sets let us obtain the following bounds. Let us notice that item (ii) establishes a result valid for every large odd prime power $q$.

Theorem 5.2. The following upper bounds hold:

(i) Let $p \geq 7$ and $q$ be prime powers such that $q=2\left(p^{2}-1\right)+1$. Then

$$
n\left(q+\sqrt{\frac{q+1}{2}}+2,5\right) \leq 2\left(q^{2}-1\right) .
$$

(ii) Given $q$ an odd prime power, let $p \geq 9$ be the highest prime power such that $q \geq 4 p^{2}-3$. Then

$$
n(q+p+2,5) \leq 2\left(q^{2}-1\right) .
$$

In particular, if $q=4 p^{2}-3$, we have

$$
n\left(q+\frac{\sqrt{q+3}}{2}+2,5\right) \leq 2\left(q^{2}-1\right) .
$$

$\operatorname{Proof}(i)$. Assume that $q=2\left(p^{2}-1\right)+1$ is a prime power for a prime power $p \geq 7$. Notice that $p=\sqrt{\frac{q+1}{2}}$. From Theorem 4.1 it follows that $n(q+p+2,5) \leq 2\left(q^{2}-1\right)$ for $p \in\{7,8,11\}$ and $q \in\{97,127,241\}$, while for $p \in\{9,16\}$ the values of $q$ are not prime powers.

For $p=13, q=337$ and $q+p+2=352$ we give the following explicit construction: Let $_{G_{L}}=S_{336}(28,56 ; 1,-1,5,-13$, $21,-43,59,67,91,-95,-121,-131,165)$ and let $G_{P}$ be the graph with $V\left(G_{P}\right)=\mathbb{Z}_{336}$ and whose edges are the ones in the graph $S_{336}(-27,-29,-23,-41,-7,-71,31,39,63,-123,-149,-159,137)$ together with the edges $\{2 i, 2 i+140\}$, for $0 \leq i \leq 167$, and the ones in the cycles $(1+2 i, 85+2 i, 197+2 i, 309+2 i, 57+2 i, 169+2 i, 281+2 i, 29+2 i, 253+$ $2 i, 141+2 i, 225+2 i, 113+2 i, 1+2 i)$, for $i=0, \ldots, 13$. Since $\omega\left(G_{P}\right) \cap \omega\left(G_{L}\right)=\emptyset$, and since the rest of suitability conditions of Theorem 2.1 are fulfilled, the graph $\mathcal{L}_{337}\left(G_{L}, G_{P}\right)$ yields $n(337+15,5) \leq 2\left(337^{2}-1\right)=227136$. Hence, item $(i)$ holds for $p \in\{7,8,11,13\}$.

From now on we assume that $p \geq 17$ and denote $N=p^{2}-1$. Let $\left\{d_{1}, \ldots, d_{p}\right\} \subset \mathbb{Z}_{p^{2}-1}$ be the relative difference set given in Lemma 5.3 and define $K=\left\{k_{1}, \ldots, k_{p}\right\}$ where $k_{i}=2 d_{i}+1 \in \mathbb{Z}_{2\left(p^{2}-1\right)}$. Consider the sets $A=\left\{k_{i}-k_{j}: 1 \leq i, j \leq p, i \neq j\right\}$, $B=\left\{k_{i}+k_{j}: 1 \leq i, j \leq p\right\}$ and $C=(2(p+1))$. Define the odd elements $m_{i} \in \mathbb{Z}_{2(p+1)}$ such that $k_{i} \equiv m_{i} \bmod 2(p+1)$. By Lemma 5.3, $C \cap A=\emptyset$ and the values $m_{i}$ are all different.

Our first step is to prove that $C \backslash B$ contains an element $\delta \neq 0$. If $\alpha=k_{i}+k_{j} \in B \cap C$ then $m_{i}+m_{j}=0$, and $\alpha$ requires a unique pair $\left\{m_{i},-m_{i}\right\}$. If $p$ is even it could be $-m_{i}=m_{i}=p+1$, but, in any case $|B \cap C| \leq\lfloor|K| / 2\rfloor=\lfloor p / 2\rfloor$. Let us take a non-zero $\delta \in C \backslash B$.

Consider the elements $P_{0}=2(p+1), Q_{0}=4(p+1), P_{1}=6(p+1), Q_{1}=8(p+1)$ in $C$ (in case $p=17$, choose $\left.Q_{1}=10(p+1)\right)$ and define the graphs $G_{L}=S_{2\left(p^{2}-1\right)}\left(P_{0}, Q_{0} ; k_{1}, \ldots, k_{p}\right)$ and $G_{P}=S_{2\left(p^{2}-1\right)}\left(P_{1}, Q_{1} ; k_{1}-\delta, \ldots, k_{p}-\delta\right)$. By Lemmas 5.1 and 2.1 this pair of graphs is suitable for amalgamation into $\mathcal{L}_{q}$ and Theorem 2.1 proves $(i)$.

(ii) Consider a prime power $p \geq 9$. As in the proof of $(i)$, define $k_{i}, B, C, \delta$ and $k_{i}^{\prime}=k_{i}-\delta$ as elements of $\mathbb{Z}_{2\left(p^{2}-1\right)}$. With abuse of language, we also denote by $k_{i}, k_{i}^{\prime} \in \mathbb{Z}$ their representatives such that

$$
-\left(p^{2}-1\right)<k_{i}, k_{i}^{\prime} \leq p^{2}-1
$$

and define the integers $P_{0}=2(p+1), Q_{0}=4(p+1), P_{1}=6(p+1), Q_{1}=8(p+1)$.

Now consider a prime power $q \geq 4 p^{2}-3$ and the graphs $G_{L}=S_{q-1}\left(P_{0}, Q_{0} ; k_{1}, \ldots, k_{p}\right)$ and $G_{P}=S_{q-1}\left(P_{1}, Q_{1} ; k_{1}^{\prime}, \ldots, k_{p}^{\prime}\right)$. From the inequalities (4),

$$
\max \left(k_{i}-k_{j}\right)<2\left(p^{2}-1\right) \leq \frac{q-1}{2} .
$$

In order to apply Lemma 2.2, it remains to see that $2 Q_{1}=16(p+1)<\frac{q-1}{2}$. A simple computation shows that this inequality holds just for $p \geq 9$ (and $q \geq 321$ ).

Moreover, from the construction of $k_{i}, k_{i}^{\prime}$, Lemma 5.1 and the inequalities (4), it follows that $G_{L}$ and $G_{P}$ share no Cayley color in $\mathbb{Z}_{q-1}$. They form a suitable pair of graphs and Theorem 2.1 provides the conclusion ( $\left.i i\right)$.

With the notations of the proof of Theorem 5.2, item $(i)$, let us notice that the graph $S_{2\left(p^{2}-1\right)}\left(k_{1}, \ldots, k_{p}\right)$ is isomorphic to the incidence graph $\mathcal{L}_{p}$. In consequence, the graphs $G_{L}, G_{P}$ are actually amalgams of 2-regular graphs into $\mathcal{L}_{p}$ and the construction in the proof has a recursive character.

Another family, defined by Ganley in [10], is the following one: Given an additive group $H$ and two subgroups $H_{1}, H_{2}$ such that $H=H_{1} \oplus H_{2}$, a direct product difference set is a subset $D=\left\{d_{1}, \ldots, d_{m}\right\} \subset H$ such that each $h \in H \backslash\left(H_{1} \cup H_{2}\right)$ is represented in a unique way as $h=d_{i}-d_{j}$, and no difference $d_{i}-d_{j}$ with $i \neq j$ lies in $H_{1} \cup H_{2}$.

Lemma 5.4 (cf. [10], Theorem 2.4). For a prime $p \geq 3$, there exists a direct product difference set $D=\left\{d_{1}, \ldots, d_{p-1}\right\}$ in the additive group $\mathbb{Z}_{p(p-1)}$ relative to the subgroups $H_{1}=(p-1)$ and $H_{2}=(p)$. 
We use these difference sets for our last result, in which we establish another bound on $n(k, 5)$.

Theorem 5.3. Let $p \geq 5$ be a prime and $q$ be a prime power such that $q=2 p(p-1)+1$. Then

$$
n\left(q+\frac{\sqrt{2 q-1}+3}{2}, 5\right) \leq 2\left(q^{2}-1\right) .
$$

Proof. For $p=5$ and $q=2 p(p-1)+1=41$, the bound $n(41+6,5) \leq 2\left(41^{2}-1\right)$ is proved in [1]. For $p=7$ and $p=11$, the result cannot be applied because $q=85$ and $q=221$ are not prime powers. Assume $p \geq 13$ is a prime. Let $d_{1}, \ldots, d_{p-1}$ be the integers provided by Lemma 5.4 and consider $k_{i}=2 d_{i}+1$. The bipartite $(p-1)$-regular graph $S_{2 p(p-1)}\left(k_{1}, \ldots, k_{p-1}\right)$ has girth $g \geq 6$.

Define sets $B=\left\{k_{i}+k_{j}: 0 \leq i, j \leq p-2\right\}$ and $C=(2(p-1)) \cup(2 p)$. Let $P_{0}=2(p-1), Q_{0}=4(p-1), P_{1}=2 p$, $Q_{1}=4 p$. Choosing a non-zero $\delta \in C \backslash B$ and following the same reasoning as in Theorem 5.2, we have that the graphs $G_{L}=S_{2 p(p-1)}\left(P_{0}, Q_{0} ; k_{1}, \ldots, k_{p-1}\right)$ and $G_{P}=S_{2 p(p-1)}\left(P_{1}, Q_{1} ; k_{1}-\delta, \ldots, k_{p-1}-\delta\right)$ are suitable for amalgamation into $\mathcal{L}_{q}$. Hence the graph $\mathcal{L}_{q}\left(G_{L}, G_{P}\right)$ has girth at least 5 , order $2\left(q^{2}-1\right)$ and degree $q+p-1+2=q+\frac{\sqrt{2 q-1}+3}{2}$.

Remark 5.1. On the one hand, the results of this section can be applied to $p=13$ and $q=313$ (Theorem 5.3), $q=337$ (Theorem 5.2, item $(i)$ ), and $q=367$ (Theorem 5.1). On the other hand, given an odd prime power $q>479$, the highest prime power $p$ such that $q \geq 4 p^{2}-3$ verifies $p \geq 11$. In this case, the bound $n(q+p+2,5) \leq 2\left(q^{2}-1\right)$ given by Theorem 5.2 is better than the result $n(q+12,5) \leq 2\left(q^{2}-1\right)$ provided by Theorem 4.1 .

The graphs constructed in this article are available in several formats. Readers can request them by email.

\section{References}

[1] E. Abajo, G. Araujo-Pardo, C. Balbuena, M. Bendala, New small regular graphs of girth 5, Discrete Math. 340 (8) (2017) $1878-1888$

[2] M. Abreu, G. Araujo-Pardo, C. Balbuena, D. Labbate, Families of small regular graphs of girth 5, Discrete Math. 312 (2012) $2832-2842$.

[3] M. Abreu, M. Funk, D. Labbate, V. Napolitano, On (minimal) regular graphs of girth 6, Australas. J. Combin. 35 (2006) $119-132$.

[4] C. Balbuena, Incidence matrices of projective planes and other bipartite graphs of few vertices, SIAM J. Discrete Math. 22 (4) (2008) $1351-1363$.

[5] J.A. Bondy, U.S.R. Murty, Graph Theory, in: Springer Series: Graduate Texts in Mathematics, vol. $244,2008$.

[6] R.C. Bose, An affine analogue of Singer's theorem, J. Indian Math. Soc. 6 (1942) 1-15.

[7] G. Exoo, Regular graphs of given degree and girth, http://ginger.indstate.edu/ge/CAGES.

[8] G. Exoo, R. Jajcay, Dynamic Cage survey, Electron. J. Combin. 15 (2008) \# DS 16, http://www.combinatorics.org/Surveys/ds16.pdf.

[9] M. Funk, Girth 5 graphs from elliptic semiplanes, Note Mat. 29 (Suppl. 1) (2009) 91-114.

[10] M.J. Ganley, Direct product difference sets, J. Combin. Theory Ser. A 23 (1977) 321-332.

[11] A.J. Hoffman, R.R. Singleton, On moore graphs with diameters 2 and 3, IBM J. (1960) 497-504.

[12] L.K. Jørgensen, Girth 5 graphs from relative different sets, Discrete Math. 293 (2005) 177-184.

[13] M. O'Keefe, P.K. Wong, A smallest graph of girth 5 and valency 6, J. Combin. Theory Ser. B 26 (1979) $145-149$.

[14] N. Robertson, The smallest graph of girth 5 and valency 4, Bull. Amer. Math. Soc. 70 (1964) 824-825.

[15] G. Royle, Cages of higher valency, http://www.cs.uwa.edu.au/ gordon/cages/allcages.html.

[16] A. Schwenk, Construction of a small regular graph of girth 5 and degree 19, in: Conference Presentation given at Normal, II, USA, 18 . April 2008.

[17] J. Singer, A theorem in projective geometry and some applications to number theory, Trans. Amer. Math. Soc. 43 (1938) $377-385$.

[18] G. Wegner, A smallest graph of girth 5 and valency 5, J. Combin. Theory Ser. B 14 (1973) 203-208.

[19] P.K. Wong, On the uniqueness of the smallest graphs of girth 5 and valency 6, J. Graph Theory 3 (1978) $407-409$.

[20] P.K. Wong, CaGes-a survey, J. Graph Theory 6 (1982) 1-22.

[21] Y.S. Yang, C.X. Zhang, A new (5, 5)-cage and the number of (5, 5)-cages (Chinese), J. Math. Res. Exposition 9 (1989) $628-632$. 